\title{
TARMED, dommages collatéraux et population civile
}

\author{
R. Leuzinger
}

\section{TARMED}

TARMED est introduit, avec plus ou moins de peine. De nombreuses imperfections sont apparues, certaines sont en voie d'amélioration. Ce tarif est conçu pour évoluer, et il faut résolument utiliser cette possibilité pour en faire un outil acceptable, équitable et accepté. Personnellement je peux m'en accommoder, les avantages de la suppression des dizaines de tarifs différents au profit d'un seul l'emportent sur les imperfections. Chaque introduction d'un nouveau tarif est laborieuse. A l'heure actuelle, je ne vois aucune alternative à TARMED.

\section{Dommages collatéraux}

Mais l'introduction de ce tarif a des effets que j'appelle des dommages collatéraux, qui ne sont pas directement liés à la structure tarifaire et dont nous n'avons, pour la plupart d'entre nous et moi y compris, pas reconnus et imaginés l'importance lorsque nous avons accepté TARMED en votation générale. Certains de ces dommages collatéraux sont déjà intervenus, d'autres menacent à court et moyen terme.

\section{Rémunération des urgences}

Malgré le fait que ce problème a rapidement été identifié et qu'une solution devrait être trouvée, il s'agit néanmoins d'un dommage collatéral important: L'idée louable de revaloriser le travail fait en urgence a été pervertie en ce sens qu'il y a des prestations tellement bien rémunérées, qu'elles sont tout bonnement inapplicables. Exemple: Les consultations téléphoniques urgentes en dehors des heures: Je n'arrive pas à envoyer une facture de plus de Fr. 100.- pour un conseil téléphonique donné après 22 heures à une personne que je n'ai jamais vu! Cette situation se produit lors de chacune de mes gardes. D'un autre côté le tarif contient une définition tellement restrictive des consultations imprévues faites pendant et en dehors des heures de consultation et qui dérangent le cours de nos journées de travail et nos nuits, que le contraire de l'idée initiale est obtenu: Nous consultons les samedis, les dimanches et les jours fériés au même tarif qu'aux heures ordinaires! Dans ces conditions, qui est encore motivé pour assumer cette tâche ingrate qu'est le service de garde?

Or, il y a de moins en moins de médecins désireux de participer aux services de garde, il faut donc les y contraindre, en introduisant dans les lois cantonales l'obligation d'y prendre part. Inutile de dire que le législateur se garde bien de prévoir une indemnisation pour ce service à la population.

\section{Transmission électronique des factures}

C'est une évidence qu'en 2004 il faut proposer d'autres systèmes de transmission des factures que ceux qui prévalaient auparavant. Mais l'acceptation de cette nécessité entraîne des effets pervers qui ne font que commencer à se faire sentir:

Afin de rendre possible cette nouvelle transmission des factures, nous avons accepté, sans nous en rendre compte, un modèle de facture type très complexe qui nécessite la récolte d'une multitude de données (numéros EAN, numéro d'assuré, numéro d'identification de chaque médicament et de chaque article médical fourni, code diagnostique, numéro d'identification d'autres fournisseurs de soins [dont nous ignorons souvent l'identité: Les patients peuvent choisir eux-mêmes tel ou tel spécialiste ou physiothérapeute], périodes d'incapacités de travail, ce qui est un renseignement totalement inutile en matière de LAMal). Le résultat est une facture de présentation absurde, composée de multiples cases, d'une multitude de rubriques, de chiffres à profusion (qui a eu la lumineuse idée d'imposer la mention des points médicaux et techniques pour chaque position tarifaire, alors que la position du tarif contient implicitement tous ces chiffres?). Ces factures sont tout bonnement illisibles pour le non-initié, alors que l'on ne cesse de promouvoir la transparence et la responsabilisation des patients aux coûts de la médecine.

Le prix en énergie, en temps et en argent nécessaire à l'adaptation de nos systèmes de gestion a été astronomique, mais c'est fait, ce dommage collatéral est déjà intervenu, nous avons encaissé 
le coup. Cet effort qu'a consenti le corps médical et les autres fournisseurs de soins aura comme effet principal de permettre aux assureurs d'introduire ces donnés dans leurs systèmes, sans devoir saisir les factures comme auparavant. Quelle économie - pour eux, mais financée par nous!

Il est à espérer que nous en tirerons, à terme, nous-mêmes un bénéfice, en disposant de nos propres statistiques, grâce aux centres de confiance. Mais l'effort que le corps médical a consenti est largement disproportionné.

D'autres dommages collatéraux découlant de ce type de facturation ne vont pas tarder à venir: Grâce à la transmission tellement détaillée de toutes les données (dont les dates, la tarification de chaque acte effectué, les codes diagnostiques), les assureurs disposeront d'un pouvoir qui leur permettra de nous dire que nous sommes trop chers (une grippe, une pneumonie, une cystite, ça coûte tant), que notre laboratoire n'est pas un laboratoire de présence (si par malheur nous facturons une analyse à une date à laquelle aucune consultation n'a eu lieu...), que nous avons travaillé trop tel ou tel jour, en additionnant les minutes de chaque prestation (est-ce qu'on nous signalera aussi quand nous ne travaillons pas assez?).

\section{Mention des diagnostics}

L'un des buts de cette mention est le calcul du coût par cas ou par diagnostic. En médecine générale, surtout chez les personnes âgées, mais aussi chez les petits enfants, la mention d'un diagnostic est illusoire: La prise en charge est presque toujours globale, avec le traitement simultané de différentes affections et de différents problèmes. Si on veut vraiment faire une analyse correcte du coût par cas, il faudra nous imposer une facturation par cas et par diagnostic! Délire de persécution? La SUVA, l'AI et l'AMF l'exigent déjà, c'est donc parfaitement réalisable... A nous d'ouvrir alors une multitude de cas parallèles, de les gérer simultanément pour une même personne pendant un même laps de temps, de les clore séparément quand le traitement d'une affection est terminé. Et cette analyse du coût par cas débouchera inévitablement sur l'établissement d'un montant jugé adéquat par diagnostic (par qui?), donc sur le reproche d'être trop cher (là aussi: serons-nous avertis si nous sommes trop bon marché?).

\section{Attestations de formation continue}

Depuis quelques années sévit la manie des certifications (ISO et autres), et nous y avons également droit, suite à une politique d'anticipation qui part de l'idée qu'il faut continuellement prouver ce que nous savons faire. Ainsi, et malgré un haut niveau de formation continue pratiquée par la grande majorité des médecins de ce pays, nous avons commencé à accepter des contrôles ou autocontrôles divers (affiliation au contrôle de qualité du laboratoire, contrôle régulier de nos installations radiologiques, attestations de formation continue). Avec l'introduction de TARMED nous avons accepté d'être désormais limités dans le choix des prestations que nous fournissons, à moins de prouver régulièrement que nous en sommes capables. Que va-t-il se produire? Suivant les règles d'application de cette obligation de certification, nous nous trouverons, d'ici peu de temps (fin 2006) face à des exigences comparables à celles qui nous sont tombés dessus avec le nouveau mode de facturation TARMED, à savoir la nécessité de dépenser beaucoup d'énergie, de temps et d'argent pour obtenir ces attestations, sous peine d'être privé $\mathrm{du}$ remboursement de ces prestations. Celles et ceux qui ont eu le douteux privilège de passer l'examen d'expert en radioprotection peuvent imaginer ce qui nous attend.

Petite parenthèse: Nos instances dirigeantes, si promptes à établir une multitude de sous-titres et de certificats de capacité, ont oublié de conditionner le droit au remboursement des prestations d'urgence à l'obtention d'un certificat de médecin de garde. Ainsi, nous avons tous obtenus, gracieusement et sans limitation dans le temps, le droit de faire la garde. Ce n'est un droit acquis pour personne, il nous est donné à vie, sans recertification aucune...

Cette méfiance sournoise envers notre profession part de la suspicion que nous ne sommes pas assez responsables pour savoir ce que nous avons appris, ce que nous savons faire et ce que nous devons perfectionner sans y être contraint. Cette obligation aura comme conséquence que nous renoncerons à fournir certaines prestations, non pas parce que nous ne savons pas les faire, mais parce qu'il devient tellement laborieux de prouver que nous savons les faire. Un autre danger guette le corps médical: Actuellement il est prévu que ce sont les sociétés de spécialistes qui fournissent ces attestations de capacité. C'est une bombe à retardement au potentiel énorme: Il suffit que les relations au sein du corps médical se détériorent, pour que cette compétence soit utilisée pour garder l'exclusivité de certaines prestations. La multiplication de ces certificats et de ces contrôles aura comme effet de réduire la palette des prestations offertes par les médecins, donc de limiter l'équipement des cabinets médicaux, surtout en périphérie. Et ce démantèlement de l'infrastructure entraînera à 
la longue, autre dommage collatéral, la disparition du métier d'assistante médicale, devenu sans attrait lorsqu'elles seront devenues exclusivement réceptionnistes et employées de bureau.

\section{Population civile}

Ce que j'appelle la population civile, ce sont les patients, mais aussi nous tous qui avons ou aurons inévitablement un jour besoin d'être soignés.

Qui est-ce qui se soucie de la population civile? Est-ce que la prise en charge de la population par le corps médical est à ce point défaillant, pour qu'il faille introduire toutes ces mesures? Est-ce que la population civile souhaite tout cela? Est-ce qu'on lui demande son avis? Les constatations et les perspectives esquissées ci-dessus auront des conséquences importantes sur le niveau des soins prodigués à la population:

- diminution de la disponibilité des médecins de garde, avec surcharge des services d'urgences des hôpitaux;

- restriction de l'offre des prestations dans les cabinets médicaux: disparition du laboratoire du praticien, de la radiologie du praticien et d'autres équipements, ce qui obligera les patients à de multiples consultations spécialisées dans les centres urbains, avec pertes de temps et de journées de travail. Le coût ainsi engendré sera énorme;

- perte de confidentialité due à la transmission des diagnostics;

- en cas de mésentente au sein du corps médical: Elle aurait inévitablement des répercus- sions néfastes sur l'efficacité et la qualité de la prise en charge pluridisciplinaire des patients;

- ces contraintes rendent la profession de moins en moins attractive, avec des conséquences sur la relève.

Sous prétexte de maîtrise des coûts, toutes sortes de décisions sont prises, des mesures sont introduites, des procédures sont créées, mais est-ce qu'on se demande en quoi tout cela améliorera la condition sanitaire de la population?

Quant au coût, il est plus que douteux que des économies puissent être réalisées grâce à ces mesures.

Il faut arrêter de compliquer les procédures (tarifaires, administratives, de formation continue) et se demander avant chaque changement, quelles en seront les conséquences pour les patients et pour le corps médical. Des procédures simples, efficaces, bien acceptées tant par les patients que par les médecins seront certainement avantageuses financièrement parlant! Les instances dirigeantes du corps médical et des assureurs doivent se rapprocher davantage des acteurs essentiels du système de santé que sont: les patients, les médecins et les autres fournisseurs de soins.

Le mécontentement des médecins grandit, la profession devient de moins en moins attractive, ce qui ne manquera pas de se répercuter négativement sur la qualité de la prise en charge de la population et la répartition judicieuse des cabinets médicaux à travers le pays. Et qui pâtira en fin de compte de cette situation? La population civile! 